\title{
Comparison of Clinical Results in Deep Vein Thrombosis of Total Knee Arthroplasty with Rivaroxaban and Dalteparin Sodium
}

\author{
Su Chan Lee, Chang Hyun Nam, Hye Sun Ahn, Seung Hyun Hwang, Nong Kyum Ahn, \\ Ha Young Park \\ Joint \& Arthritis Research, Department of Orthopaedic Surgery, Himchan Hospital, Seoul, Korea \\ Email: changcape@naver.com
}

Received 18 January 2015; accepted 9 February 2015; published 11 February 2015

Copyright (C) 2015 by authors and Scientific Research Publishing Inc.

This work is licensed under the Creative Commons Attribution International License (CC BY). http://creativecommons.org/licenses/by/4.0/

(c) (i) Open Access

\section{Abstract}

This study was intended to investigate into the incidence rates of deep vein thrombosis (DVT) in patients who used prophylactic antithrombotic medications after total knee arthroplasty (TKA), and to compare clinical results in groups treated with Rivaroxaban versus Dalteparin sodium as prophylactic antithrombotic medications. This prospective study was performed in 300 patients who underwent TKA between November 2011 and December 2012. The prophylactic therapy was given to 150 patients in Rivaroxaban group and Dalteparin sodium group, respectively. In addition, intermittent compression pump and stocking were used in all the groups immediately after TKA. In order to determine the incidence of DVT, color Doppler ultrasonography, D-dimer, and clinical symptom examination were conducted. There were 17 cases $(11.3 \%)$ of DVT in Rivaroxaban group and 18 cases $(12.0 \%)$ of DVT in Dalteparin sodium group after TKA, and no significant difference was seen between both groups. Between patients with DVT and those without DVT after TKA at 4 days in both groups, there was a significant difference in the swelling indices. Moreover, a significant difference was observed in the evaluation of bruise. The early signs of DVT after TKA are unknown, however, some initial clinical signs such as swelling have been observed. After using the said prophylactic drugs, the lower incidence of DVT was seen, and there was no difference between the types of drugs. Pharmacological therapy (either Rivaroxaban or Dalteparin sodium) after TKA is considered effective for DVT prevention. There is also a need to consider constant monitoring of clinical symptoms.

\section{Keywords}

Deep Vein Thrombosis, Total Knee Arthroplasty, Rivaroxaban, Dalteparin Sodium 


\section{Introduction}

As South Korea is becoming an aging society, a growing number of elderly people suffer from diseases. As a typical degenerative disease, degenerative arthritis usually has great influence on the patient's daily life due to its severe pain. For this reason, some patients with early knee osteoarthritis take medication and physiotherapy that can help to reduce pains, and the number of patients with end-stage knee osteoarthritis undergoing total knee arthroplasty (TKA) is on the rise. Accordingly, complications following TKA are actively discussed and studied.

Especially, DVT is a relatively common complication in orthopedic surgery, and in severe cases, it causes a pulmonary embolism. The clinical features of DVT are non-specific and asymptomatic despite some symptoms such as pain, swelling, heat, tenderness or redness in the lower leg. Thus, early detection and prevention of DVT are critically important.

Some studies reported that when prophylaxis was not used, $40 \%$ - $80 \%$ of the patients developed DVT and $0.5 \%-2 \%$ developed fatal pulmonary embolism [1]. However, a combination of two prophylaxes-mechanical thromboprophylaxis (i.e., compression stockings, early walking, and/or intermittent pneumatic compression pump) and pharmacological thromboprophylaxis (i.e., aspirin, low molecular weight heparin, waparin, and/or dextran) have reduced the incidence rates to $15 \%-30 \%$ in the Western countries [2] [3].

Kim et al. reported that the importance of preoperative prophylaxis has not been emphasized owing to $10 \%$ $20 \%$ of low incidence rates. Nevertheless, up to $40 \%$ of incidence rates are currently reported along with the steadily increasing frequency in the Eastern countries [4].

In the past few years, several new oral anticoagulants have been developed. Most of these drugs focus on directly inhibiting either Factor Xa (Rivaroxaban, Apixaban, and Edoxaban) or thrombin (Dabigatran). The benefits of these drugs include easy administration (oral versus intravenous or subcutaneous), no need for routine monitoring, and improved safety profiles [5]-[7].

Rivaroxaban has been approved as a treatment option indicated for DVT prevention after some orthopedic surgical procedures in the United States, Canada and Europe. Also, in September 2011, the US FDA approved Rivaroxaban for the reduction of stroke and thrombosis in atrial fibrillation patients as well as for the prevention of thrombosis in patients undergoing total knee or hip arthroplasty. A rise in demand for the said medication was also observed in South Korea. In 2011, Patel, M.R., et al. reported that there was no statistically significant difference in stroke, systemic embolism, major bleeding and non-major bleeding among Rivaroxaban, direct factor Xa inhibitor, and warfarin. Rivaroxaban reduced intracranial and fatal bleeding, compared with warfarin [8].

Dalteparin sodium, another prophylactic antithrombotic medication administered subcutaneously, has the advantage of relatively low drug interactions, and thus obviates the need to monitor blood coagulation. In addition, it has been widely used in clinical practice by virtue of well-established efficacy and stability [3].

In this context, the present study examined the clinical results of medical therapies after TKA, and then compared the clinical results between the two groups treated with either Rivaroxaban (Xarelto ${ }^{\circledR}$ ) or Dalteparin so$\operatorname{dium}\left(\right.$ Fragmin $\left.^{\circledR}\right)$.

\section{Materials and Methods}

This study was approved by the Hospital's Ethical Committee at our institution, and all participants were provided with informed consent (ICF). We prospectively evaluated the data from 300 patients who underwent primary TKA from November 2011 to December 2012. They consisted of 38 men (12.7\%) and 262 women (87.3\%) with the mean age and body mass index (BMI) of 69.1 years $( \pm 6.9)$ and $26.2 \mathrm{~kg} / \mathrm{m}^{2}( \pm 3.6)$, respectively. Their etiologies included degenerative arthritis (290 cases), osteonecrosis (8 cases) and rheumatoid arthritis (2 cases). The number of patients receiving either unilateral TKA or bilateral TKA (with an one-week interval) was 156 and 144, respectively. In bilateral TKA cases, the first surgery only was examined. The patients all used Scorpio NRG (Non-Restrictive Geometry, Stryker ${ }^{\circledR}$, Allendale, NJ, USA). Also, the TKA was performed under spinal anesthesia by a single surgeon. The patients were evenly divided into the Rivaroxaban group and Dalteparin sodium group, and each group with 150 patients showed similar distribution (Table 1).

The dose and duration of medication were prescribed correctly according to the guidelines of the pharmaceutical companies. When used as prophylaxis, Rivaroxaban is recommended to be used for 14 days, and Dalteparin sodium to be used for 5 days [9]-[11]. Accordingly, Rivaroxaban $10 \mathrm{mg}$ was orally administered in Rivaroxaban group once daily from Day 1 to Day 14 after TKA, where as Dalteparin sodium was subcutaneously 
Table 1. Demographic and clinical characteristic of patients.

\begin{tabular}{|c|c|c|c|}
\hline & Rivaroxaban $\left(\right.$ Xarelto $\left.^{\circledR}\right)$ & Dalteparin Sodium $\left(\right.$ Fragmin $^{\circledR}$ ) & p-value \\
\hline Patients, n & 150 & 150 & \\
\hline Age, yr (range) & $68.7(51-88)$ & $69.6(58-86)$ & 0.280 \\
\hline Gender, M:F & $16: 134$ & $22: 128$ & 0.298 \\
\hline $\mathrm{BMI}^{\mathrm{a}}, \mathrm{kg} / \mathrm{m}^{2}$ (range) & $26.2(17-37)$ & $26.1(14-34)$ & 0.940 \\
\hline Unilateral TKA, n (P/C $)$ & $51(34.0 \%)$ & $105(70.0 \%)$ & \multirow{2}{*}{0.513} \\
\hline Bilateral TKA, n (P/C $\left.{ }^{b}\right)$ & $99(66.0 \%)$ & $45(30.0 \%)$ & \\
\hline Diabetes, $\mathrm{n}\left(\mathrm{P} / \mathrm{C}^{\mathrm{b}}\right)$ & $33(22.0 \%)$ & $33(22.0 \%)$ & 1.000 \\
\hline Hypertension, $\mathrm{n}\left(\mathrm{P} / \mathrm{C}^{\mathrm{b}}\right)$ & $102(67.3 \%)$ & $99(66.0 \%)$ & 0.713 \\
\hline Hyperlipidemia, $\mathrm{n}\left(\mathrm{P} / \mathrm{C}^{\mathrm{b}}\right)$ & $46(30.7 \%)$ & $38(25.3 \%)$ & 0.304 \\
\hline Smoking, $n\left(P / C^{b}\right)$ & $1(0.7 \%)$ & $1(0.7 \%)$ & 1.000 \\
\hline History of lower limb surgery, $\mathrm{n}\left(\mathrm{P} / \mathrm{C}^{\mathrm{b}}\right)$ & 35 (23.3\%) & 36 (24\%) & 0.491 \\
\hline
\end{tabular}

${ }^{\mathrm{a}} \mathrm{BMI}$ : body mass index; ${ }^{\mathrm{b}} \mathrm{P} / \mathrm{C}$ : percentage.

administered in Dalteparin sodium group at a dose of 5000 IU once daily from Day 1 to Day 5 after TKA. In addition, both intermittent compression pump and compression stocking were used in all the groups immediately after TKA, and knee joint exercise was performed using continuous passive motion (CPM) at Days 2 and 3 after TKA.

To analyze the correlation with risk factors affecting the incidence of DVT, demographic and clinical characteristics of patients including age, gender, BMI, diabetes, hypertension, hyperlipidemia, smoking and the history of lower limb surgery were examined. The clinical symptoms of DVT were examined at Days 4 and 9 after TKA. During examination of clinical symptoms, the severity of distal femoral pain and proximal tibial pain was assessed using a verbal rating scale (VRS) ( 0 = no pain; 1 = mild pain; 2 = moderate pain; and, 3 = severe pain) [7]. Thigh swelling was assessed by measuring the circumference of the thigh at a distance of $10 \mathrm{~cm}$ from the patella in both the lower limbs with and without the surgical site, and subsequently categorizing differences between the measurements into four grades ( $0=$ no swelling; $1=$ under $1.5 \mathrm{~cm} ; 2=1.6-3.0 \mathrm{~cm}$; and, $3=3 \mathrm{~cm}$ or more) [12]. We measured respective factors on the distal femur and proximal tibia. The severity of thigh bruising was assessed on the basis of the criteria proposed by Warwick et al. [13] [14]. Specifically, it shall be 0 point for no color change; 1 point for light yellow; 2 points for dark yellow; 3 points for yellow in the area of at least three palms; 4 points for yellow-black; and, 5 points for yellow-black in the area of at least three palms. In addition, local heat around the patella was assessed and adjudicated as one of mild, moderate or severe in consideration for the patient's gait, exercise and fomentation just before examination of clinical symptoms, followed by checking the patient for a positive Homan's sign.

At Day 7 after TKA, the same radiologist examined the common femoral vein, superficial femoral vein, and popliteal vein using color Doppler ultrasound imaging to determine the presence of DVT.

The results of each group were expressed as mean, standard deviation and frequency (\%), and statistical analyses of respective parameters were conducted using SPSS (18.0 for Windows, Chicago, Illinois) statistical software program. Risk factors seen in the two groups were analyzed with independent t-test at a significance level of $p<0.05$.

\section{Results}

\subsection{Incidence of DVT}

There were 17 (11.3\%, 17/150) cases of DVT in Rivaroxaban group and 18 (12.0\%, 18/150) cases of DVT in Dalteparin sodium group after TKA. In Rivaroxaban group, there were $6(11.8 \%)$ cases of DVT out of 51 patients who underwent unilateral TKA, and 11 (11.1\%) cases of DVT out of 99 patients who underwent bilateral TKA, and no statistically significant difference was shown. In Dalteparin sodium group, the proportions of pa- 
tients developing DVT after the unilateral or bilateral TKA were 12.7\% and 11.5\%, respectively, and no statistically significant difference was observed. No statistically significant difference was observed in both groups, depending on average age, gender, and BMI. Additionally, no statistically significant difference was found in terms of diabetes, hypertension, hyperlipidemia, and smoking status that are generally considered as risk factors of DVT $(p>0.05)$ (Table 2).

\subsection{Clinical Symptom Examination}

The indexes of distal femoral pain in DVT and non-DVT patients were 1.1 and 0.8 in Rivaroxaban group. The index of proximal tibial pain was 1.5 in the DVT patients, which was close to light pain value, while it was 1.2 in the non-DVT patients. In Dalteparin sodium, the indexes of distal femoral pain in DVT and non-DVT patients were 1.1 and 1.0, respectively while those of proximal tibial pain observed were 1.2 and 0.8 , respectively, indicating no statistically significant difference (Table 3 and Table 4).

In Rivaroxaban group, the indexes of distal femoral swelling in DVT and non-DVT patients were 1.6 and 1.1, respectively at Day 4 after TKA, suggesting a statistically significant difference between the two patient groups $(p=0.014)$. Also, the indexes of proximal tibial swelling observed were 1.8 and 1.3 , respectively, suggesting a statistically significant difference between the two patient groups $(p=0.001)$. In Dalteparin sodium group, the indexes of distal femoral swelling in DVT and non-DVT patients were 1.5 and 1.3, respectively, indicating a statistically significant difference between the two patient groups $(p=0.042)$. Additionally, the index of proximal tibial swelling was 1.7 in DVT patients, and 1.0 in non-DVT patients, thus implying a statistical significant difference (Table 3 and Table 4).

In Rivaroxaban group, the indexes of bruise in DVT and non-DVT patients were 1.9 and 1.4 which were close to dark yellow when the seriousness of bruise was assessed at Day 4 after TKA ( $p=0.024)$. In Dalteparin sodium group, the indexes of bruise were 2.5 and 1.7 in DVT and non-DVT patients, respectively $(p=0.077)$. Further, no statistically significant difference in bruise was observed between the two patient groups in Dalteparin sodium group that previously showed a significant difference (Table 3 and Table 4).

In Rivaroxaban group, the indexes of local heat sensation were equivalent to 1.1 and 1.1 in DVT and nonDVT patients, respectively. In Dalteparin sodium group, the local heat sensation indexes were 1.4 and 1.1, respectively, which suggests no statistically significant difference.

The results of all the clinical symptoms, except for swelling, examined at Day 9 after TKA show no statistically significant difference in Dalteparin sodium group.

Table 2. Comparison of general factors between two groups.

\begin{tabular}{|c|c|c|c|c|c|c|}
\hline & \multicolumn{3}{|c|}{ Rivaroxaban (Xarelto ${ }^{\circledR}$ ) } & \multicolumn{3}{|c|}{ Dalteparin Sodium $\left(\right.$ Fragmin $^{\circledR}$ ) } \\
\hline & Positive & Negative & $p$-value & Positive & Negative & p-value \\
\hline Patients, n (P/C $\left.{ }^{a}\right)$ & $17(11.3 \%)$ & $133(88.7 \%)$ & & $18(12.0 \%)$ & $132(88.0 \%)$ & \\
\hline Age, yr (range) & $68(51-88)$ & $69(53-88)$ & 1.000 & $69(61-77)$ & $70(58-86)$ & 0.822 \\
\hline Gender, M:F & $4: 13$ & $12: 121$ & 0.068 & $4: 14$ & 18:114 & 0.334 \\
\hline $\mathrm{BMI}^{\mathrm{b}}, \mathrm{kg} / \mathrm{m}^{2}$ (range) & $25(17-31)$ & $26(18-37)$ & 0.156 & $26(14-32)$ & $26(16-34)$ & 0.990 \\
\hline Unilateral TKA, n (P/C $)$ & $6(11.8 \%)$ & $45(88.2 \%)$ & \multirow{2}{*}{0.905} & $8(12.7 \%)$ & $55(87.3 \%)$ & \multirow{2}{*}{0.154} \\
\hline Bilateral TKA, n (P/C $)$ & $11(11.1 \%)$ & $88(88.9 \%)$ & & $10(11.5 \%)$ & 77 (88.5\%) & \\
\hline Diabetes, n (P/C $\left.{ }^{a}\right)$ & $2(11.8 \%)$ & $31(23.3 \%)$ & 0.279 & $3(16.7 \%)$ & $30(22.7 \%)$ & 0.560 \\
\hline Hypertension, $\mathrm{n}\left(\mathrm{P} / \mathrm{C}^{\mathrm{a}}\right)$ & $9(52.9 \%)$ & $93(69.9 \%)$ & 0.157 & $11(61.1 \%)$ & $88(66.7 \%)$ & 0.641 \\
\hline Hyperlipidemia, n (P/C ) & $3(17.6 \%)$ & $43(32.3)$ & 0.216 & $3(16.7 \%)$ & 35 (26.5\%) & 0.367 \\
\hline Smoking, $\mathrm{n}\left(\mathrm{P} / \mathrm{C}^{\mathrm{a}}\right)$ & $0(0.0 \%)$ & $1(0.8 \%)$ & 0.720 & $0(0.0 \%)$ & $1(0.8 \%)$ & 0.711 \\
\hline History of lower limb surgery, $n\left(\mathrm{P} / \mathrm{C}^{\mathrm{a}}\right)$ & $4(23.5 \%)$ & $31(23.3 \%)$ & 0.768 & 7 (38.9\%) & $29(22.0 \%)$ & 0.214 \\
\hline
\end{tabular}

${ }^{\mathrm{a} P} / \mathrm{C}$ : percentage; ${ }^{\mathrm{b}} \mathrm{BMI}$ : body mass index. 
Table 3. Univariate analysis results for clinical symptoms in Rivaroxaban group.

\begin{tabular}{|c|c|c|c|c|c|c|c|}
\hline & & \multicolumn{3}{|c|}{ POD\#4 } & \multicolumn{3}{|c|}{ POD\#9 } \\
\hline & & DVT (+) n (\%) & DVT (-) n (\%) & p-value & DVT (+) n (\%) & DVT (-) n (\%) & p-value \\
\hline & 0 & $2(11.8)$ & 35 (26.3) & & $14(82.4)$ & 102 (76.7) & \\
\hline & 1 & $11(64.7)$ & $88(66.2)$ & & $3(17.6)$ & $30(22.6)$ & \\
\hline Distal femur & 2 & $4(23.5)$ & $10(7.5)$ & 0.066 & $0(0.0)$ & $1(0.8)$ & 0.837 \\
\hline & 3 & $0(0.0)$ & $0(0.0)$ & & $0(0.0)$ & $0(0.0)$ & \\
\hline & Index & 1.1 & 1.8 & & 0.2 & 0.2 & \\
\hline \multicolumn{8}{|l|}{ Pain } \\
\hline & 0 & $0(0.0)$ & $21(15.8)$ & & $10(58.8)$ & 77 (57.9) & \\
\hline & 1 & $9(52.9)$ & 68 (51.1) & & $7(41.2)$ & 55 (41.4) & \\
\hline Proximal tibia & 2 & $8(47.1)$ & 44 (33.1) & 0.17 & $0(0.0)$ & $1(0.8)$ & 0.937 \\
\hline & 3 & $0(0.0)$ & $0(0.0)$ & & $0(0.0)$ & $0(0.0)$ & \\
\hline & Index & 1.5 & 1.2 & & 0.4 & 0.4 & \\
\hline & 0 & $0(0.0)$ & 18 (13.5) & & $11(64.7)$ & $111(83.5)$ & \\
\hline & 1 & $8(47.05)$ & $86(64.7)$ & & $6(35.3)$ & $22(16.5)$ & \\
\hline Distal femur & 2 & $8(47.05)$ & 21 (15.8) & $0.014^{*}$ & $0(0.0)$ & $0(0.0)$ & 0.062 \\
\hline & 3 & $1(5.9)$ & $8(6.0)$ & & $0(0.0)$ & $0(0.0)$ & \\
\hline & Index & 1.6 & 1.1 & & 0.4 & 0.7 & \\
\hline \multicolumn{8}{|l|}{ Swelling } \\
\hline \multirow{5}{*}{ Proximal tibia } & 0 & $0(0.0)$ & $1(0.8)$ & & $8(47.1)$ & 79 (59.4) & \\
\hline & 1 & $5(29.4)$ & $91(68.4)$ & & $8(47.1)$ & 53 (39.8) & \\
\hline & 2 & $11(64.7)$ & $41(30.8)$ & $0.001^{* *}$ & $1(5.9)$ & $1(0.8)$ & 0.168 \\
\hline & 3 & $1(5.9)$ & $0(0.0)$ & & $0(0.0)$ & $0(0.0)$ & \\
\hline & Index & 1.8 & 1.3 & & 0.6 & 0.4 & \\
\hline \multirow{7}{*}{ Bruising } & 0 & $0(0.0)$ & 13 (9.8) & & 12 (70.6) & 98 (73.7) & \\
\hline & 1 & $7(41.2)$ & 77 (57.9) & & $4(23.5)$ & 33 (24.8) & \\
\hline & 2 & $7(41.2)$ & 21 (15.8) & & $1(5.9)$ & $2(1.5)$ & \\
\hline & 3 & $1(5.9)$ & 18 (13.5) & $0.024^{*}$ & $0(0.0)$ & $0(0.0)$ & 0.478 \\
\hline & 4 & $2(11.8)$ & $4(3.0)$ & & $0(0.0)$ & $0(0.0)$ & \\
\hline & 5 & $0(0.0)$ & $0(0.0)$ & & $0(0.0)$ & $0(0.0)$ & \\
\hline & Index & 1.9 & 1.4 & & 0.4 & 0.3 & \\
\hline \multirow{5}{*}{ Local heat } & 0 & $0(0.0)$ & 13 (9.8) & & 15 (88.2) & 105 (78.9) & \\
\hline & 1 & 15 (88.2) & 93 (69.9) & & $2(11.8)$ & 27 (20.3) & \\
\hline & 2 & $2(11.8)$ & 27 (20.3) & 0.231 & $0(0.0)$ & $1(0.8)$ & 0.651 \\
\hline & 3 & $0(0.0)$ & $0(0.0)$ & & $0(0.0)$ & $0(0.0)$ & \\
\hline & Index & 1.1 & 1.1 & & 0.1 & 0.8 & \\
\hline
\end{tabular}

Significantly different between the two groups, ${ }^{*}=p$-value $<0.05 ;{ }^{* *}=p$-value $<0.001 ;{ }^{* * *}=p$-value $<0.0001$. 
Table 4. Univariate analysis results for clinical symptoms in Dalteparin sodium group.

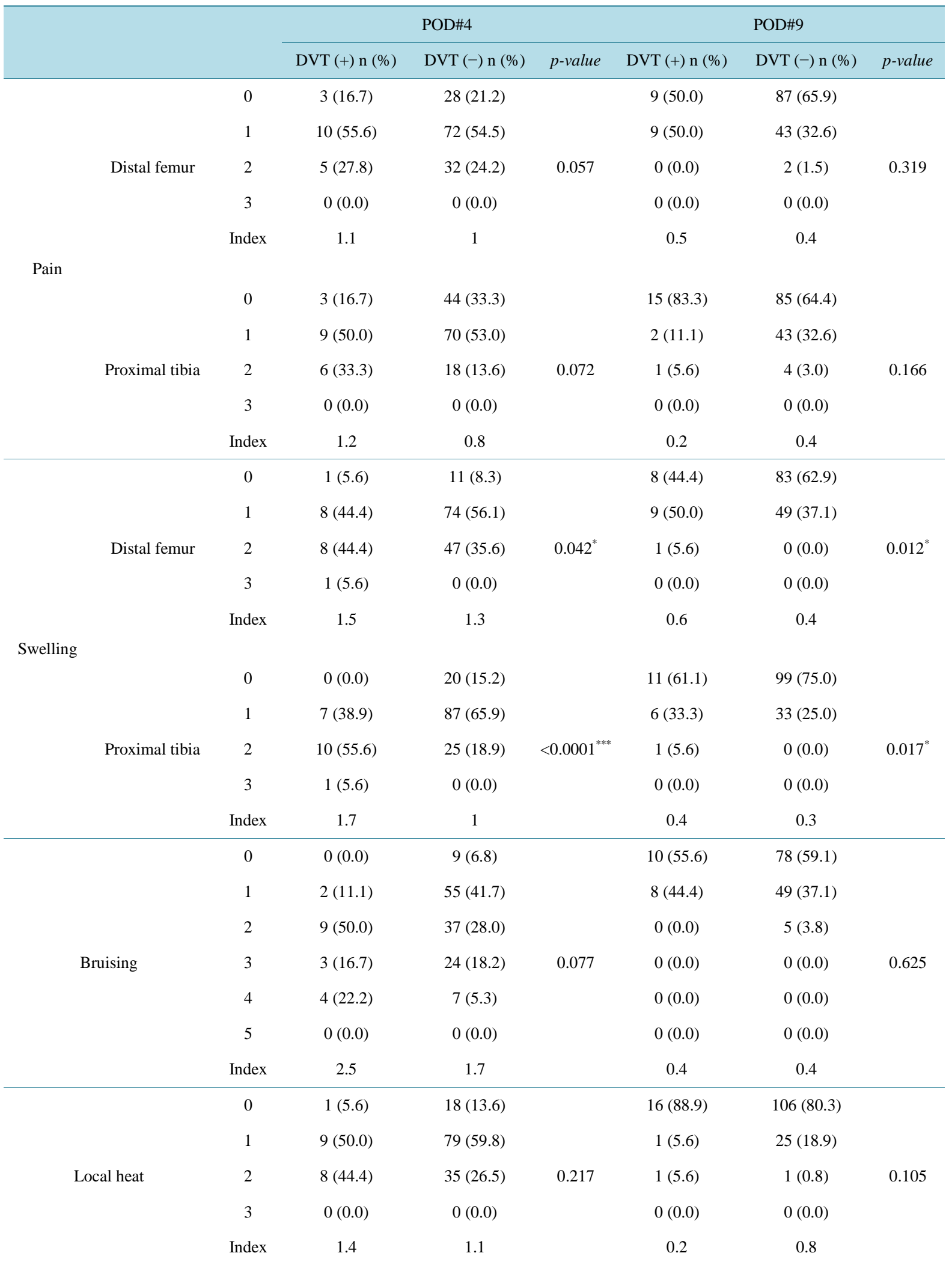

Significantly different between the two groups, ${ }^{*}=p$-value $<0.05 ;{ }^{* *}=p$-value $<0.001 ;{ }^{* * *}=p$-value $<0.0001$. 


\section{Discussion}

The rate of post-TKA complications, composed primarily of ones associated with revision TKA, is estimated at $23.6 \%$ and is considered very high in recent years. Although the uncertain cause of DVT after TKA is complex and multifactorial, lower limb surgery itself may be one of the main risk factors of DVT.

In this study, prophylactic medication was used to treat DVT after TKA, and then the clinical results were compared between Rivaroxaban group and Dalteparin sodium group. The incidence rate of DVT was $11.3 \%$ in Rivaroxaban group and $12.0 \%$ in Dalteparin sodium group, indicating that the incidence rate was $0.7 \%$ lower in Rivaroxaban group. However, no statistically significant difference was observed, and there was also no case of pulmonary thromboembolism in both the groups.

TKA itself is a risk factor of DVT because it may cause venous congestion, excessive clotting, and/or blood vessel intimal injury. In addition, many studies suggest that it is associated with such risk factors as age, gender, obesity, varicosity, hypertension, diabetes, mobility, and/or the duration of tourniquet use, though their relationship remains unclear [15] [16]. In this study, any statistically significant difference in these factors was not found between DVT and non-DVT patients.

DVT is difficult to predict its onset, and it is critically important to give timely treatments through early diagnosis. Therefore, it is crucial to carefully and closely monitor the postoperative clinical symptoms.

In this study, it was observed in Rivaroxaban group that the indexes of distal femoral swelling and proximal tibial swelling were significantly higher in DVT patients than in non-DVT patients at Day 4 after TKA. Similarly, in Dalteparin sodium group, the indexes of distal femoral swelling and proximal tibial swelling were higher in DVT patients than in non-DVT patients at Day 4 after TKA. When the severity of bruise was evaluated, no significant difference was observed in Dalteparin sodium group. By contrast, in Rivaroxaban group, the bruise indexes were 1.9 and 1.4 in DVT and non-DVT patients at Day 4 after TKA, and showed a statistically significant difference. Furthermore, there was no significant difference between DVT and non-DVT patients in other clinical symptoms, such as local heat sensation around the patella and the pains in the distal femur/proximal tibia. Also, at Day 9 after TKA, no significant difference in swelling and the degree of bruise was seen.

This study has the following limitations. First, we did not use venography for DVT diagnosis. Venography is a gold standard for DVT diagnosis, however this technique is invasive and requires the use of potentially hazardous contrast agents [17]. Since it might not be suitable immediately after TKA, we diagnosed DVT using color Doppler ultrasound imaging. Second, in spite of some studies reporting the delayed events of post-TKA DVT at up to Day 30, this study assessed only early events of DVT up to Day 10 [18] [19]. Nevertheless, the clinical symptoms and outcomes of DVT were carefully followed up in visits at Months 1 and 3, and no case of DVT was found. Finally, no comparison was made in terms of the dose and treatment duration of the prophylactic drugs administered. As this study was focused on the prophylactic effects of the two drugs, rather than the comparison in terms of dose and usage, it was deemed that there would be no decisive influence on the purpose of this study. However, it is considered that further studies need to be conducted with respect to the prophylactic effects on DVT based on dose and usage.

Following the prophylactic therapy after TKA, it was found that the incidence rate of DVT was $11.3 \%$ in Rivaroxaban (Xarelto ${ }^{\circledR}$ ) group and $12.0 \%$ in Dalteparin sodium (Fragmin ${ }^{\circledR}$ ) group which shows that the incidence rate was $0.7 \%$ lower in Rivaroxaban $\left(\right.$ Xarelto $^{\circledR}$ ) group, however no statistically significant difference was observed. There was neither event of DVT nor DVT-induced complication in all patients at Day 9 after TKA. There is also a need to take into account the constant monitoring of clinical symptoms in patients at high risk of thrombosis (that is, having thrombosis related risk factors). In conclusion, a pharmacological therapy (Rivaroxaban or Dalteparin sodium) after TKA is considered effective for DVT prevention.

\section{References}

[1] Lieberman, J.R. and Geerts, W.H. (1994) Prevention of Venous Thromboembolism after Total Hip and Knee Arthroplasty. Journal of Bone and Joint Surgery (America), 76, 1239-1250. http://jbjs.org/content/76/8/1239.long

[2] Geerts, W.H., Bergqvist, D., Pineo, G.F., Heit, J.A., Samama, C.M., Lassen, M.R. and Colwell, C.W., American College of Chest Physicians (2008) Prevention of Venous Thromboembolism: American College of Chest Physicians Evidence-Based Clinical Practice Guidelines (8th Edition). Chest, 133, 381S-453S.

[3] Arcelus, J.I., Kudrna, J.C. and Caprini, J.A. (2006) Venous Thromboembolism Following Major Orthopedic Surgery: What Is the Risk after Discharge? Orthopedics, 29, 506-516. http://dx.doi.org/10.1080/028418501127346846 
[4] Kim, Y.H. and Kim, J.S. (2002) Incidence and Natural History of Deep-Vein Thrombosis after Total Knee Arthroplasty. A Prospective, Randomised Study. Journal of Bone and Joint Surgery (British), 84, 566-570. http://dx.doi.org/10.1302/0301-620X.84B4.12330

[5] Little, J.W. (2012) New Oral Anticoagulants: Will They Replace Warfarin? Oral Surgery, Oral Medicine, Oral Pathology and Oral Radiology, 113, 575-580. http://dx.doi.org/10.1016/j.0000.2011.10.006

[6] Agnelli, G. (2004) Prevention of Venous Thromboembolism in Surgical Patients. Circulation, 110, IV4-IV12. http://dx.doi.org/10.1161/01.CIR.0000150639.98514.6c

[7] Duggan, S.T., Scott, L.J. and Plosker, G.L. (2009) Rivaroxaban: A Review of Its Use for the Prevention of Venous Thromboembolism after Total Hip or Knee Replacement Surgery. Drugs, 69, 1829-1851. http://dx.doi.org/10.2165/11200890-000000000-00000

[8] Patel, M.R., Mahaffey, K.W., Garg, J., Pan, G., Singer, D.E., Hacke, W., Breithardt, G., Halperin, J.L., Hankey, G.J., Piccini, J.P., Becker, R.C., Nessel, C.C., Paolini, J.F., Berkowitz, S.D., Fox, K.A. and Califf, R.M., ROCKET AF Investigators (2011) Rivaroxaban versus Warfarin in Nonvalvular Atrial Fibrillation. The New England Journal of Medicine, 365, 883-891. http://dx.doi.org/10.1056/NEJMoa1009638

[9] Mavrakanas, T. and Bounameaux, H. (2011) The Potential Role of New Oral Anticoagulants in the Prevention and Treatment of Thromboembolism. Pharmacology \& Therapeutics, 130, 46-58. http://dx.doi.org/10.1016/j.pharmthera.2010.12.007

[10] Pfizer Medical Information (2012) Fragmin (Dalteparin Sodium) Abbreviated Prescribing Information All Presentations and Indications. Pfizer Inc. http://www.medisis.com/client_docs/fragmin/fragmin_pi.pdf

[11] Levine, M., Gent, M., Hirsh, J., Leclerc, J., Anderson, D., Weitz, J., Ginsberg, J., Turpie, A.G., Demers, C. and Kovacs, M. (1996) A Comparison of Low-Molecular-Weight Heparin Administered Primarily at Home with Unfractionated Heparin Administered in the Hospital for Proximal Deep-Vein Thrombosis. The New England Journal of Medicine, 334, 677-681. http://dx.doi.org/10.1056/NEJM199603143341101

[12] Song, E.K., Seon, J.K., Park, S.J., Cho, S.B. and Choi, M.S. (2008) Diagnosis of the Deep Vein Thrombosis with Multidetector-Row Computed Tomographic Venography after Total Knee Arthroplasty. The Journal of the Korean Orthopaedic Association, 43, 294-300. http://dx.doi.org/10.4055/jkoa.2008.43.3.294

[13] Warwick, D., Harrison, J., Glew, D., Mitchelmore, A., Peters, T.J. and Donovan, J. (1998) Comparison of the Use of a Foot Pump with the Use of Low-Molecular-Weight Heparin for the Prevention of Deep-Vein Thrombosis after Total Hip Replacement. A Prospective, Randomized Trial. Journal of Bone and Joint Surgery-American Volume, 80, 11581166.

[14] Lee, J.K, Chun, K.S., Baek, S.W. and Choi, C.H. (2012) The Prophylaxis of Venous Thromboembolism in Korean Patients with Total Knee Replacement Arthroplasty. The Journal of the Korean Orthopaedic Association, 47, 86-95. http://dx.doi.org/10.4055/jkoa.2012.47.2.86

[15] Kim, Y.H. and Suh, J.S. (1988) Low Incidence of Deep-Vein Thrombosis after Cementless Total Hip Replacement. Journal of Bone and Joint Surgery-American Volume, 70, 878-882.

[16] Sharrock, N.E., Haas, S.B., Hargett, M.J., Urquhart, B., Insall, J.N. and Scuderi, G. (1991) Effects of Epidural Anesthesia on the Incidence of Deep-Vein Thrombosis after Total Knee Arthroplasty. Journal of Bone and Joint Surgery-American Volume, 73, 502-506.

[17] Carpenter, J.P., Holland, G.A., Baum, R.A., Owen, R.S., Carpenter, J.T. and Cope, C. (1993) Magnetic Resonance Venography for the Detection of Deep Venous Thrombosis: Comparison with Contrast Venography and Duplex Doppler Ultrasonography. Journal of Vascular Surgery, 18, 734-741. http://dx.doi.org/10.1016/0741-5214(93)90325-G

[18] Dahl, O.E., Gudmundsen, T.E. and Haukeland, L. (2000) Late Occurring Clinical Deep Vein Thrombosis in Joint-Operated Patients. Acta Orthopaedica, 71, 47-50. http://dx.doi.org/10.1080/00016470052943883

[19] Douketis, J.D., Eikelboom, J.W., Quinlan, D.J., Willan, A.R. and Crowther, M.A. (2002) Short-Duration Prophylaxis against Venous Thromboembolism after Total Hip or Knee Replacement: A Meta-Analysis of Prospective Studies Investigating Symptomatic Outcomes. Archives of Internal Medicine, 162, 1465-1471.

http://dx.doi.org/10.1001/archinte.162.13.1465 
Scientific Research Publishing (SCIRP) is one of the largest Open Access journal publishers. It is currently publishing more than 200 open access, online, peer-reviewed journals covering a wide range of academic disciplines. SCIRP serves the worldwide academic communities and contributes to the progress and application of science with its publication.

Other selected journals from SCIRP are listed as below. Submit your manuscript to us via either submit@scirp.org or Online Submission Portal.
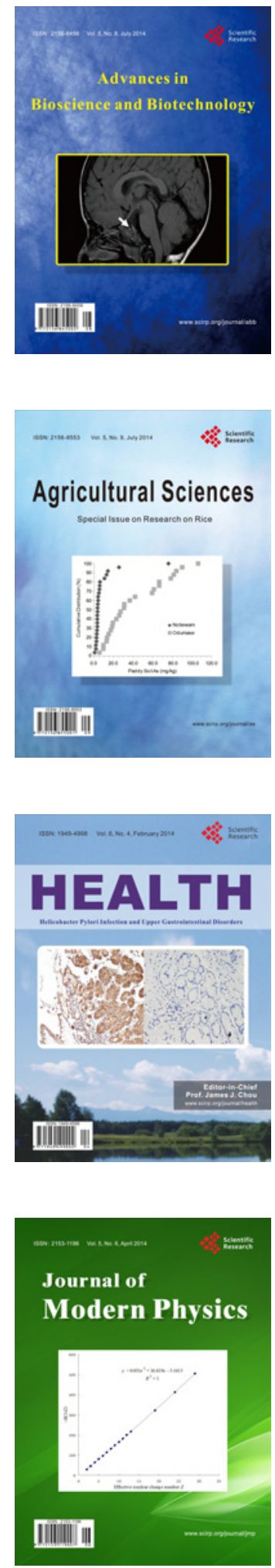
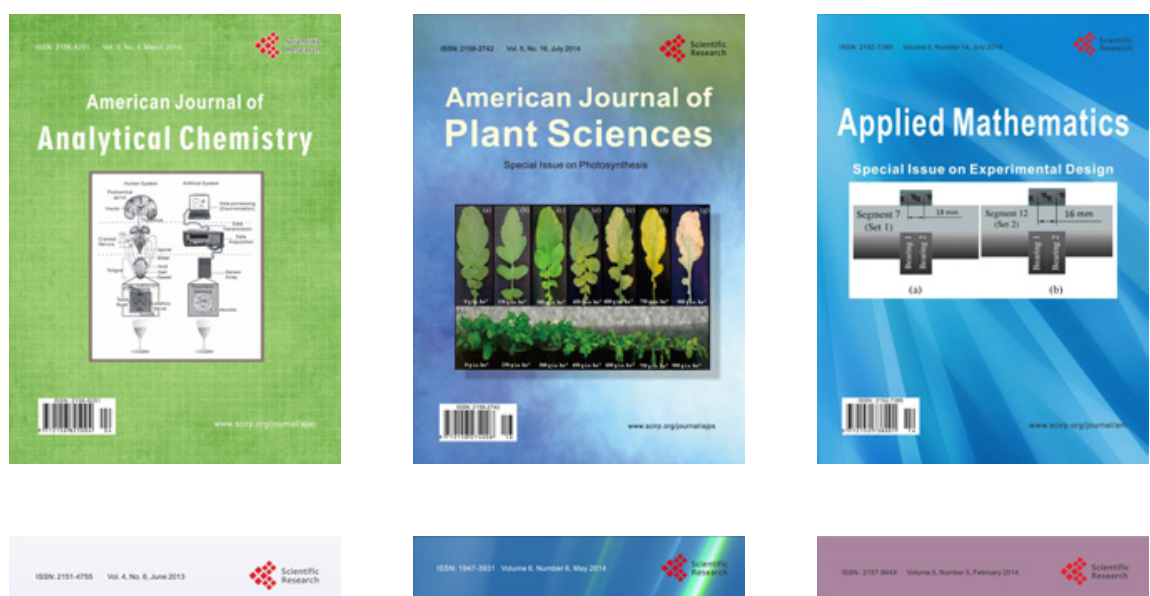

Creative Education
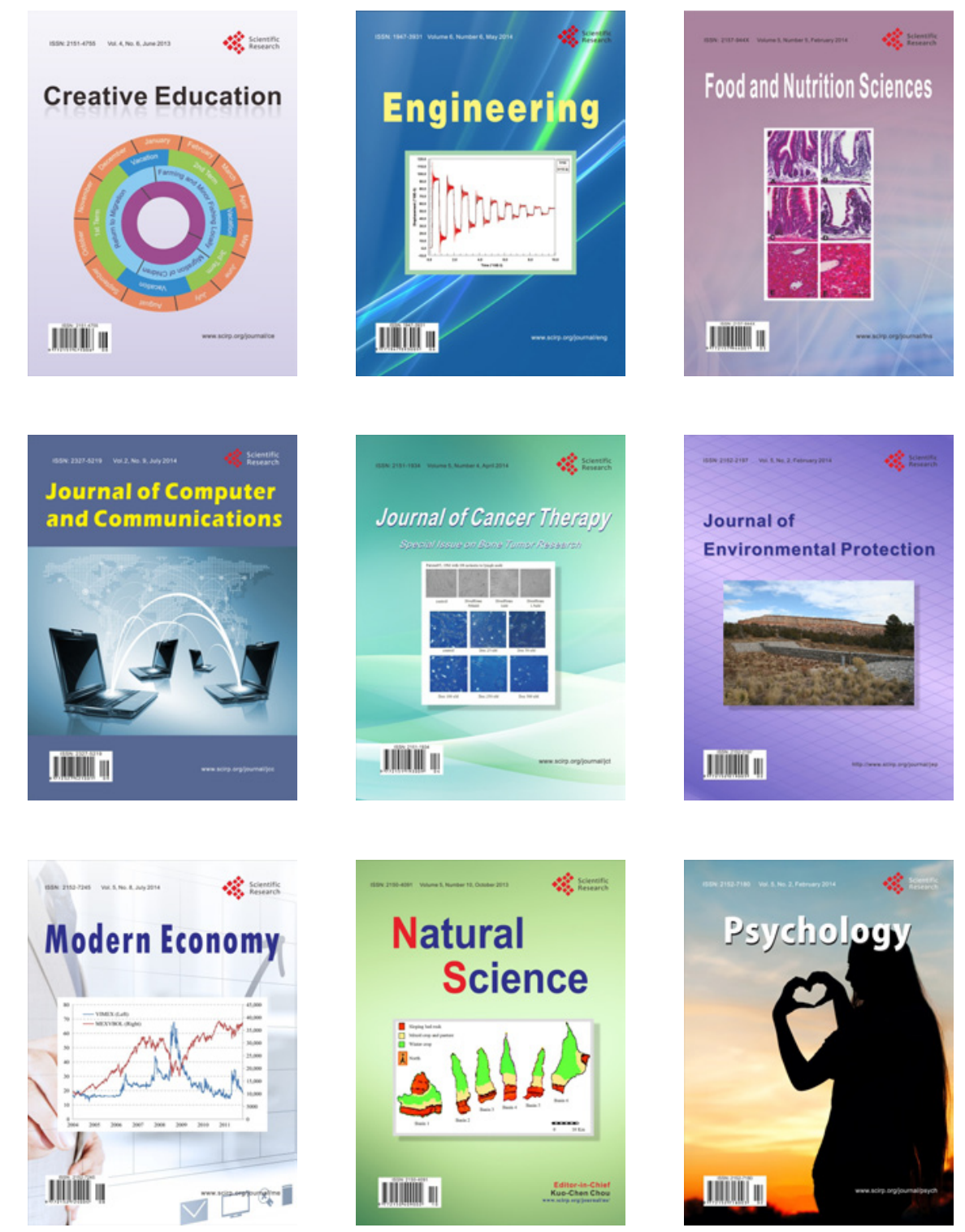